\title{
A bio-inspired celestial compass applied to an ant-inspired robot for autonomous navigation*
}

\author{
Julien Dupeyroux $^{1}$, Julien Diperi ${ }^{1}$, Marc Boyron ${ }^{1}$, Stéphane Viollet $^{1}$ and Julien Serres ${ }^{1}$
}

\begin{abstract}
Common compass sensors used in outdoor environments are highly disturbed by unpredictable magnetic fields. This paper proposes to get inspiration from the insect navigational strategies to design a celestial compass based on the linear polarization of ultraviolet (UV) skylight. This bioinspired compass uses only two pixels to determine the solar meridian direction angle. It consists of two UV-light photosensors topped with linear polarizers arranged orthogonally to each other as it was observed in insects' Dorsal Rim Area. The compass is embedded on our ant-inspired hexapod walking robot called Hexabot. The performances of the celestial compass under various weather and UV conditions have been investigated. Once embedded onto the robot, the sensor was first used to compensate for yaw random disturbances. We then used the compass to maintain Hexabot's heading direction constant in a straight-forward walking task over a flat terrain while being perturbated in yaw by its walking behaviour. Experiments under various meteorological conditions provided steady state heading direction errors from $0.3^{\circ}$ (clear sky) to $1.9^{\circ}$ (overcast sky). These results suggest interesting precision and reliability to make this new optical compass suitable for autonomous field robotics navigation tasks.
\end{abstract}

\section{INTRODUCTION}

Autonomous navigation systems mostly make use of Global Positioning System (GPS) and Inertial Measurement Units (IMU). Unfortunately, the precision of GPS methods is in the range of one meter, which makes them not suitable for all robotic applications. Besides, local magnetic fields produced by ferrous materials in urban infrastructures can cause incorrect magnetometer measurements. These magnetic disturbances are classically compensated applying Kalman filtering and require data fusion from gyroscopes and accelerometers [1]. The solutions currently proposed remain sensitive to multiple sources of disturbances since gyroscopes and accelerometers are also prone to drifts. Biomimetic approach has led to interesting models for optimization, control and automation in robotics. In complex outdoor environments where magnetic interferences are increasingly present and often unpredictable, bio-inspired solutions would be interesting to abstract from magnetic pollution and GPS precision.

We propose here to get inspiration from the insects' skylight polarization compass [2] to provide a reliable measurement of the heading direction for outdoor robotic tasks. The scattering of sunlight within the Earth's atmosphere

*This work was supported by the French Direction Générale de l'Armement (DGA), CNRS, Aix-Marseille Université, the Provence-AlpesCôte d'Azur region and the French National Research Agency for Research (ANR) with the Equipex/Robotex project.

${ }^{1}$ Aix-Marseille University, CNRS, ISM, Inst Movement Sci, Marseille, France. julien. serresduniv-amu. fr produces a polarization pattern across the sky. Solar radiations remain unpolarized until their entry into the atmosphere where scattering interactions with atmospheric constituents induce the partially linear polarization of the skylight [3]. The direction of the linear polarization of skylight at the zenith point is always perpendicular to the solar meridian ${ }^{1}$. The angle direction of the solar meridian is not constant and highly depends on the position of the Earth about the sun and the location of the observer.

Biological studies showed the existence of ommatidia sensitive to the polarization of skylight in the Dorsal Rim Area (DRA) of the insect's compound eye ([4], [5]). Each ommatidium is sensitive to a unique direction of polarization and its orthogonal direction, and the spectral sensitivity is generally in the ultraviolet (UV) range, except for a few species whose maximal sensitivity is in the blue range or in the green range. Many hypotheses have been proposed to explain why ommatidia are sensitive to UV light instead of other spectral ranges, but the most reasonable assumption is that the skylight polarization remains strong in the UV under canopies and clouds ([6], [7]). In the locust brain, the information perceived through the DRA is first integrated by polarization neurons, called POL-neurons, in the optic lobe, which shows a high synaptic activity for three distinct orientations $\left(10^{\circ}, 60^{\circ}\right.$ and $\left.130^{\circ}\right)$, while in the central complex, POL-neurons show a rather uniform synaptic activity for all polarization angles [8]. DRA-based neural models mostly provide an estimated heading direction by computing the logarithmic difference between the response of the ommatidium to a single polarization orientation, and the response of the same ommatidium to the corresponding orthogonal polarization orientation [5]. The study of the DRA in desert ants and honeybees showed that insects refer to a unique global polarization angle [9] to get their bearings. For instance, during a foraging trip in an unknown place, desert ants Cataglyphis integrate their heading direction through their celestial compass. Although their foraging trip consists of a slightly random exploration trajectory, their homing trajectory tends to be direct and straight to the nest [9].

The first autonomous wheeled robot to use a celestial compass, Sahabot 1, was created by Lambrinos et al. in the late 1990s [10]. Getting inspiration from the DRA of the cricket, the spectral sensitivity ranged from $400 \mathrm{~nm}$ to $520 \mathrm{~nm}$. The project sought to test three models of heading direction measurement: (a) the scanning model uses only

\footnotetext{
${ }^{1}$ The solar meridian is the circle that crosses the zenith and the sun relative to an observer on the Earth.
} 
one polarization sensor, consisting of a pair of photodiodes mounted below orthogonally set linear polarizer, and makes the robot rotate to find angle that provides the highest sensor response. However, the rotating phase often induces 2D displacements and therefore increase the final position error in a navigational task; (b) the extended scanning model uses the same procedure as the scanning model but with three polarization sensors set at different orientations $\left(0^{\circ}, 60^{\circ}\right.$ and $120^{\circ}$ ) as in the optic lobe of insects. The solar meridian direction is then computed simply by subtracting the sensor signals. This method provides more reliable results since peaks detected at the corresponding linear polarization angle are sharper than in the scanning model, but 2D drift remains an issue; (c) the simultaneous model uses three polarization sensors without rotating the robot. Logarithmic differences are computed between each sub-unit of POL-sensors so that the heading direction can be correctly estimated. Tests were performed in the early morning and the average angular error was of $0.66^{\circ}$ using the simultaneous model, and $1.73^{\circ}$ using the simple scanning model. The simultaneous model was then applied to Sahabot 2 in order to implement antinspired path integration models [11]. It is still unclear how insects distinguish solar and anti-solar angles, but some suggest that insects use a circadian clock to both dispel the heading direction ambiguity and compensate the sun path [12]. Sahabot simply integrated the position of the sun to avoid any ambiguity ([10], [11]).

Chu et al. developed a celestial compass based on the one integrated in the Sahabot projects, using the simultaneous model ([13], [14]). The optical compass was embedded onto a wheeled robot using a fuzzy logic controller to follow a preprogrammed trajectory. Tests were performed at the end of the day to prevent any sensors saturation. A miniaturized version of the celestial compass has also been proposed [15] but no implementation onto a mobile robot has been recorded yet. Another implementation of the celestial compass has been embedded on a small Unmanned Aerial Vehicle (UAV) [16]. Three polarization sensors, including effective directions and their corresponding orthogonal, were integrated in an ocelli based autopilot designed to control the UAV roll and pitch over ten seconds of flight [16].

In this paper, we propose to merge both scanning and simultaneous models proposed by Lambrinos et al. into a UV-polarized light scanning model providing highly accurate measurement of the heading direction of our walking robot under various meteorological conditions and a low UVindex ${ }^{2}$. Section II presents the UV-polarized light compass and analyzes its performance under highly different weather and UV conditions. Section III describes the hexapod walking robot. Section IV examines two practical experiments of heading direction recovery using our celestial compass in real outdoor conditions.

\footnotetext{
${ }^{2}$ Experiments conducted with Sahabot were done in desert conditions whith high UV range (UV-index of 11 in Maharès, Tunisia, in August 1996). Most of the time, the sky was clear. Source: http://www.temis.nl/uvradiation/UVindex.html
}

\section{THE UV-POLARIZED LIGHT COMPASS}

\section{A. The 3D-printed UV-polarized light sensor}

The celestial compass uses two UV-light sensors mounted below rotating UV linear sheet polarizers held by two 70teeth gears (see figure 1.B) which are driven by a third one composed of 10 teeth and actuated by a stepper motor (AM0820-A-0,225-7, Faulhaber). Due to its symmetric properties, the two UV sheet polarizers holder gears turn in the same direction. The entire prototype was printed using PLA filament (polyactic acid). The angular resolution of the compass can be modified by changing the microstep settings of the stepper motor. The UV-light sensor is SG01D-18 (SgLux) which active area is equal to $0.5 \mathrm{~mm}^{2}$ and spectral sensitivity is between $200 \mathrm{~nm}$ and $375 \mathrm{~nm}$ with a maximum spectral response at $280 \mathrm{~nm}$. Each POL-unit has an angular field of view of $\pm 50^{\circ}$, and a refresh rate of $33.3 \mathrm{~Hz}$. The UV linear sheet polarizer has a local maximum single (resp. parallel) UV-light transmission of $52 \%$ (resp. $27 \%$ ) for wavelengths between $270 \mathrm{~nm}$ and $400 \mathrm{~nm}$. The peak transmission is located near $330 \mathrm{~nm}$.

We call POL-sensor any sub-unit of the compass composed of a UV-light photo-receptor topped with a UV sheet polarizer. The left (resp. right) POL-sensor is called $U V_{0}$ (resp. $U V_{1}$ ). Let $x$ be the rotation angle of the UV sheet polarizer holder gears, and $\psi$ the solar meridian direction angle. Therefore, the response of each POL-sensor unit is:

$$
\left\{\begin{array}{c}
U V_{0}(x)=A_{0}+B_{0} \cdot \cos (2(x+\psi)) \\
U V_{1}(x)=A_{1}+B_{1} \cdot \cos \left(2\left(x+\psi+\frac{\pi}{2}\right)\right)
\end{array}\right.
$$

where $x \in[0 ; 2 \pi]$ is the angle of rotation of the polarizers and $\psi \in[0 ; \pi]$ is the solar meridian direction angle, $A_{0}$ and $A_{1}$ are offsets determined by the average UV-light radiance and inner bias of each photo-sensor, $B_{0}$ and $B_{1}$ are constants determined by the degree of polarization and inner gain of each photo-sensor, and $U V_{0}$ and $U V_{1}$ are $\pi$-periodic sinusoidal functions. POL-sensors measurements are normalized so that the minimum value is set at 0 and the maximum value is set at 1 . In case of bad weather conditions, $B_{0}$ and $B_{1}$ values are significantly reduced due to the weakening of the degree of polarization, implying heavy noise disturbances in POLsensors measurements. To prevent incorrect computation of $\psi$, we propose to restrict the signal to its first harmonic. Let $U V_{0}^{n c}$ (resp. $U V_{1}^{n c}$ ) be the normalized and corrected $U V_{0}$ (resp. $U V_{1}$ ) POL-sensor measurement:

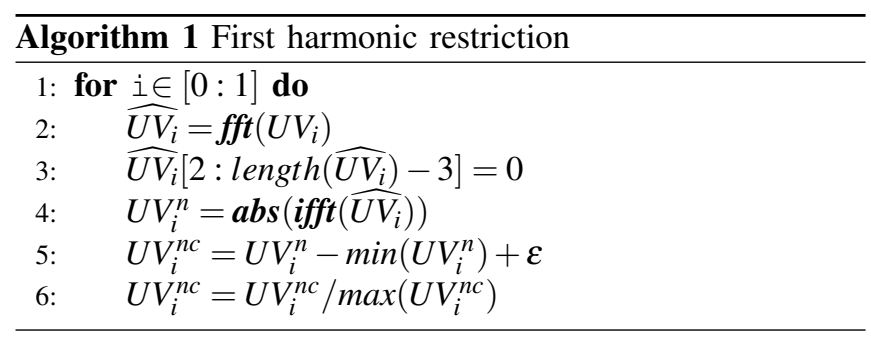

where fft and ifft are respectively the direct and reverse fast Fourier transforms, and $\varepsilon=0.0001$ is set to prevent 


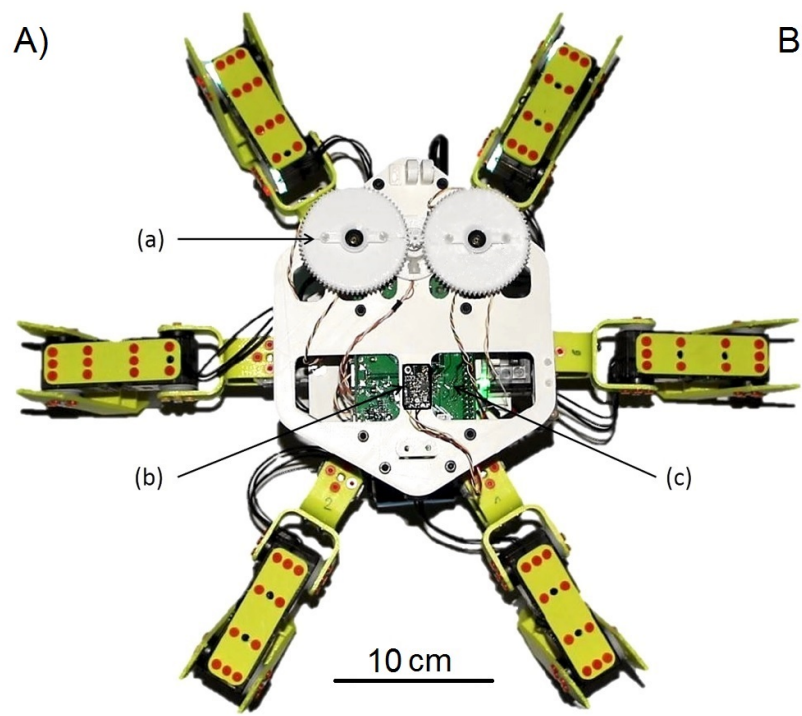

B)

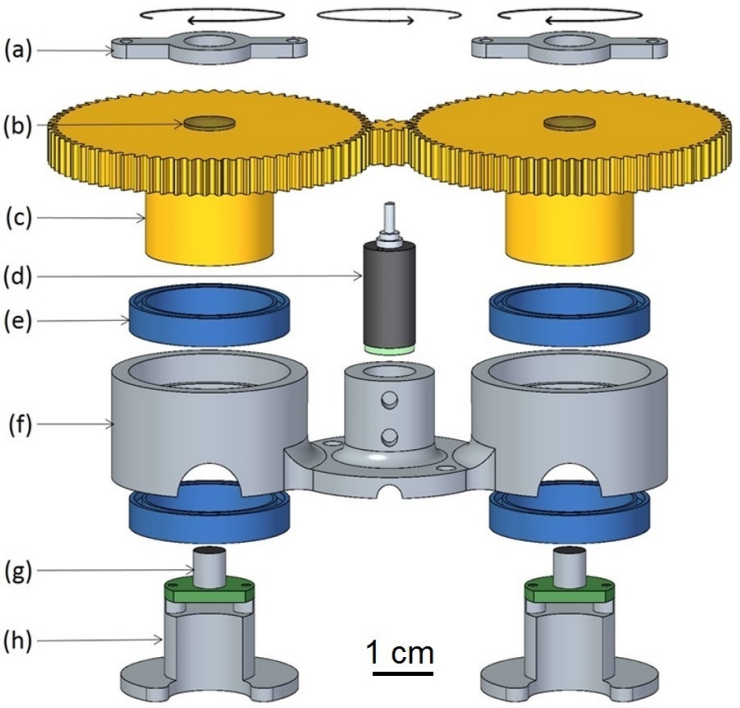

Fig. 1. A. Top view of the Hexabot robot equipped with the UV-polarized light compass. (a) Celestial compass; (b) MinImu-9 v.3 gyro, accelerometer \& compass (Pololu) used for outdoor ground truth measurement; (c) Raspberry Pi 2B board. B. An exploded view of the compass. (a) 3D-printed fixation (PLA, polyactic acid) for the UV sheet polarizer; (b) UV linear sheet polarizer (HNP'B replacement, UV grade 275 - 750nm); (c) 3D-printed gears (PLA); (d) stepper motor AM0820-A-0,225-7 (Faulhaber); (e) ball bearing; (f) 3D-printed support (PLA); (g) UV-light sensor SG01D-18 (SgLux); (h) 3D-printed support (PLA) for UV-light sensor.

from logarithm calculation failure. The POL-unit response is defined as follows :

$$
p(x)=\log _{10}\left(\frac{U V_{1}^{n c}(x)}{U V_{0}^{n c}(x)}\right) .
$$

We then compute the solar meridian direction $\psi$ by locating the two local minimum values of the $p$ function, the first one being in $[0 ; \pi]$ and the second one in $[\pi ; 2 \pi]$ :

$$
\psi=\frac{1}{2}(\underset{x \in[0 ; \pi]}{\arg \min } p(x)+\underset{x \in[\pi ; 2 \pi]}{\arg \min } p(x)-\pi) .
$$

due to the symmetry of the polarization pattern around the zenith point, $\psi$ is only known within $[0 ; \pi]$. Classical methods to eliminate the ambiguity between $\psi_{\text {Solar }}$ and $\psi_{\text {Anti-Solar }}$ use the ambient radiance distribution. As none of the tasks asked from the robot imply a turn back movement, there was no reason for $\psi$ to change for $\psi+\pi$. Therefore, we assume that $\psi \in[0 ; \pi]$. Using the average value of the two minima of function $p$ provides more accuracy in determining the solar meridian direction angle $\psi$ relative to the robot.

\section{B. Performances of the celestial compass under various weather conditions}

The celestial compass was tested under various weather conditions in order to quantify its reliability and performances in determining the solar meridian angular direction. Four sets of data obtained in February and April 2017 under both clear and covered sky conditions are shown in figure 2. The UV-index was equal to 1 in February, and 7 in April according to the French meteorological services.

The table I provides the average peak-to-peak magnitudes $\overline{U V_{0, P-P}}$ (resp. $\overline{U V_{1, P-P}}$ ) of raw signals $U V_{0, k}$ (resp. $U V_{1, k}$ ), and the corresponding coefficient of variation $C v_{p}$, where $k \in \llbracket 1 . . n \rrbracket$ stands for the $k$-th test, $n$ is the number of tests conducted, and $p \in\{0,1\}$ corresponds to the left and right POL-units. Letters standing for conditions are used with respect to the identifiers of graphs in figure 2.

TABLE I

PEAK-TO-PEAK MAGNITUDE OF RAW SIGNALS

\begin{tabular}{cccccc} 
Conditions & $\overline{U V_{0, P-P}}$ & $C v_{0}$ & $\overline{U V_{1, P-P}}$ & $C v_{1}$ & $n$ \\
\hline (a,b) & 333.19 & $6 \%$ & 396.00 & $6 \%$ & 21 \\
$(\mathrm{c}, \mathrm{d})$ & 79.47 & $22 \%$ & 124.93 & $22 \%$ & 15 \\
$(\mathrm{e}, \mathrm{f})$ & 959.06 & $5 \%$ & 1137.11 & $5 \%$ & 36 \\
$(\mathrm{~g}, \mathrm{~h})$ & 176.11 & $18 \%$ & 111.22 & $21 \%$ & 36 \\
\hline
\end{tabular}

The estimated cosine waves $U V_{0}^{n c}$ and $U V_{1}^{n c}$ were compared to the normalized raw signals by applying the Mean Squared Error (MSE) method. Therefore, the error $\varepsilon_{p, k}$ is defined as

$$
\varepsilon_{p, k}=\operatorname{MSE}\left(U V_{p, k}^{n c}\right)=\frac{1}{N} \sum_{i=1}^{N}\left(U V_{p, k}^{n c}(i)-U V_{p, k}^{n}(i)\right)^{2}
$$

where $N$ is the total length of the signal. Due to some improvements made in the stepper-motor control, $N$ got risen from 280 in February to 373 in April. Finally, in table II we compute the average error $\overline{\varepsilon_{p}}$ as the mean value of all $\varepsilon_{p, k}$ for $k \in \llbracket 1 . . n \rrbracket$. The coefficients of variation $C v\left[\varepsilon_{p}\right]$ were also calculated.

The overall results show that it is clearly impossible to distinguish whether the estimation of the angular direction $\psi$ was done in winter or not, and under clear sky or not. We notice that both average errors and coefficients of variation are highly similar between February and April at a given weather condition. Besides, though the variability of signals is heavily increased under covered sky, the celestial compass 


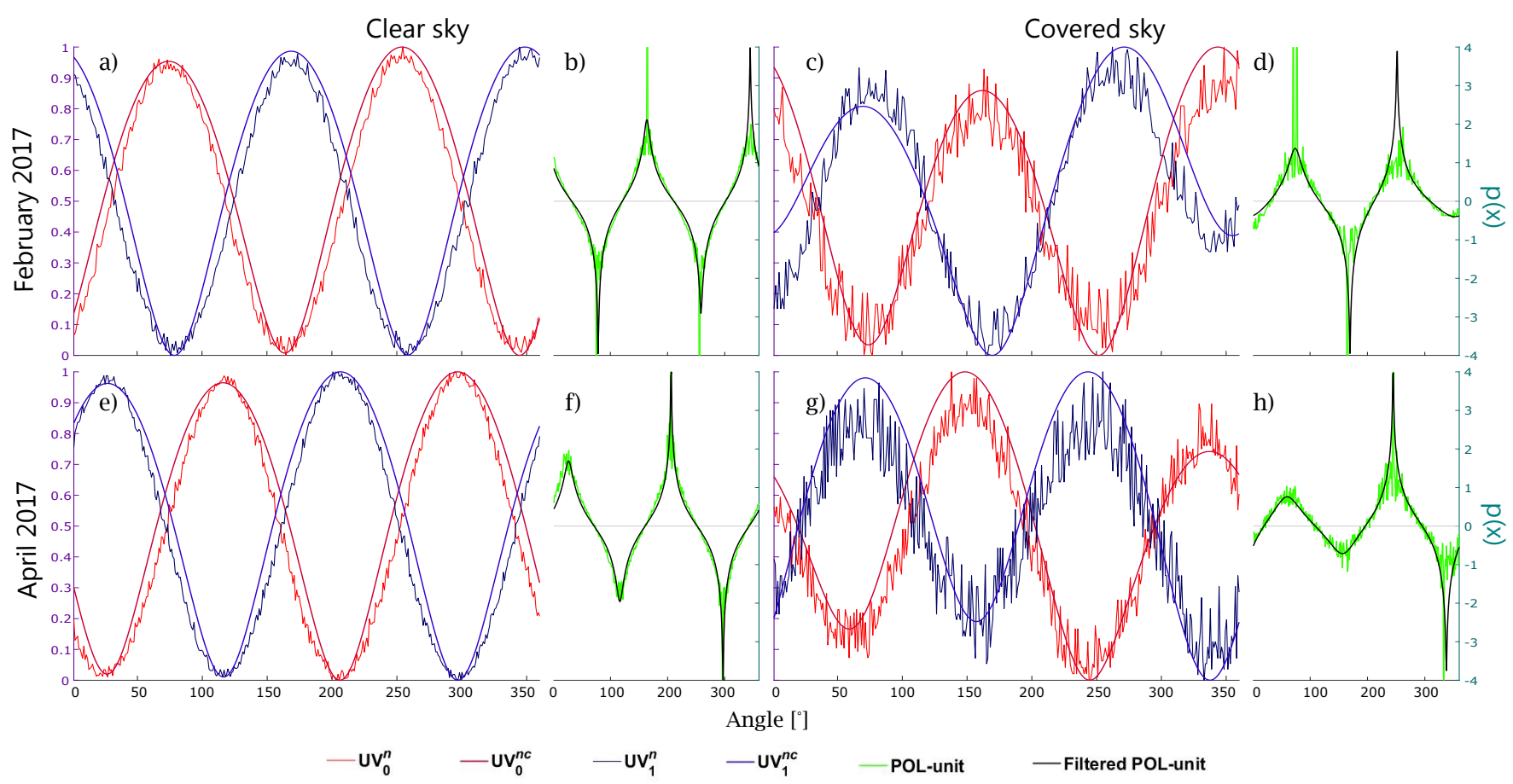

Fig. 2. Examples of signals obtained during several acquisitions from the UV-polarized light compass. Each pairs of graphs (a,b), (c,d), (e,f) and (g,h) show normalized raw and filtered outputs for $U_{0}$ and $U_{V}$ photo-sensors (left), and the POL-unit response for both raw and filtered data(right). Pair (a,b) was obtained in February 2017 under clear sky conditions with a UV index equal to 1, pair (c,d) at the same period but under covered sky. Pairs (e,f) and (g,h) were obtained in April 2017 with a UV index of 7 - the first one under clear sky, the second one under covered sky.

TABLE II

STEADY STATE ERROR BETWEEN NORMALIZED AND FILTERED DATA

\begin{tabular}{cccccc} 
Conditions & $\overline{\varepsilon_{0}}$ & $C v\left[\varepsilon_{0}\right]$ & $\overline{\varepsilon_{1}}$ & $C v\left[\varepsilon_{1}\right]$ & $n$ \\
\hline$(\mathrm{a}, \mathrm{b})$ & $4.28 \mathrm{e}-03$ & $6 \%$ & $4.83 \mathrm{e}-03$ & $4 \%$ & 21 \\
$(\mathrm{c}, \mathrm{d})$ & $9.02 \mathrm{e}-03$ & $36 \%$ & $7.31 \mathrm{e}-03$ & $32 \%$ & 15 \\
$(\mathrm{e}, \mathrm{f})$ & $3.99 \mathrm{e}-03$ & $10 \%$ & $4.14 \mathrm{e}-03$ & $5 \%$ & 36 \\
$(\mathrm{~g}, \mathrm{~h})$ & $6.14 \mathrm{e}-03$ & $27 \%$ & $8.36 \mathrm{e}-03$ & $19 \%$ & 36 \\
\hline
\end{tabular}

remains fully able to accurately estimate $\psi$. The restriction of the signal to its first harmonic is consequently an interesting approach to provide a good estimate of the solar meridian angle direction $\psi$ without introducing delay.

\section{HEXABOT : THE ANT-INSPIRED ROBOT}

\section{A. The robot platform}

A fully open source, 3D-printed, six-legged walking robot called Hexabot ${ }^{3}$ (figure 3) has been developped to mimic the desert ant in several navigational tasks such as homing in unknown environments. The overall weight of Hexabot, including batteries, is $925 \mathrm{~g}$, and the maximum length is $360 \mathrm{~mm}$ with a maximum height of the center of mass is $145 \mathrm{~mm}$. Its battery endurance, depending on the capacity, ranges from half an hour to one hour. The robot has three DYNAMIXEL XL-320 actuators per leg which allows to reach high walking speed (approximately $35 \mathrm{~cm} / \mathrm{s}$ in optimal conditions) and execute complex motion when crossing over an uneven terrain. Besides, six-legged robots show more stable walking motion than the four-legged ones since they

${ }^{3}$ Based on Metabot, a quadruped walking robot. See http://metabot.cc/ can operate static gait (i.e. three to five legs remain on the ground at any time).

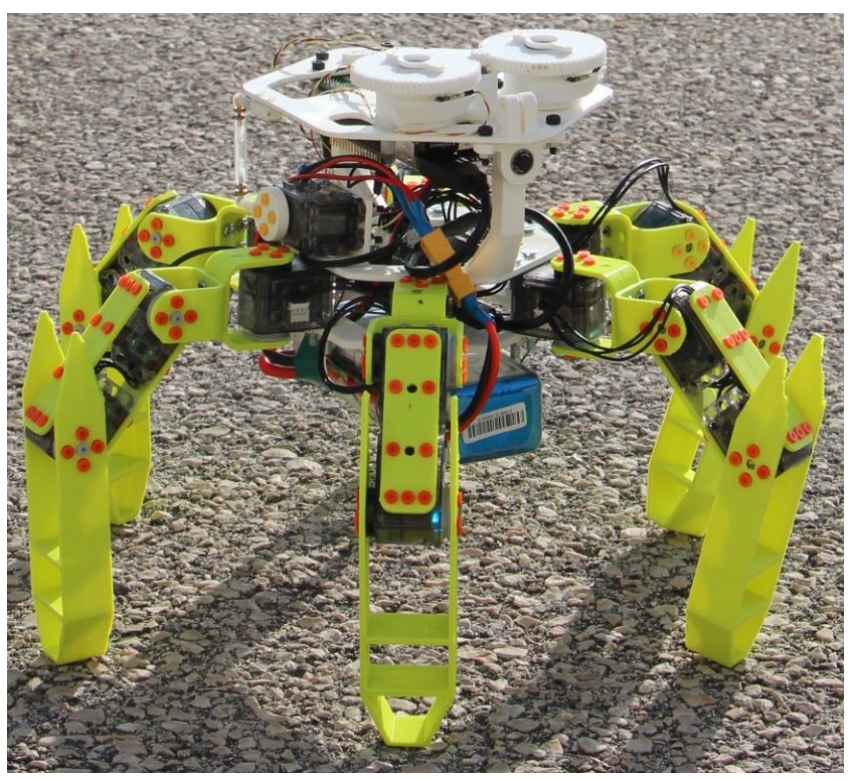

Fig. 3. Hexabot robot equiped with a pair of UV-polarized light sensors forming a celestial compass.

\section{B. Robot electronic architecture}

An OpenCM9.04C micro-controller (32-bit ARM CortexM3) controls the robot. This first board is connected to a second one, a Raspberry Pi 2B board (32-bit quad-core 


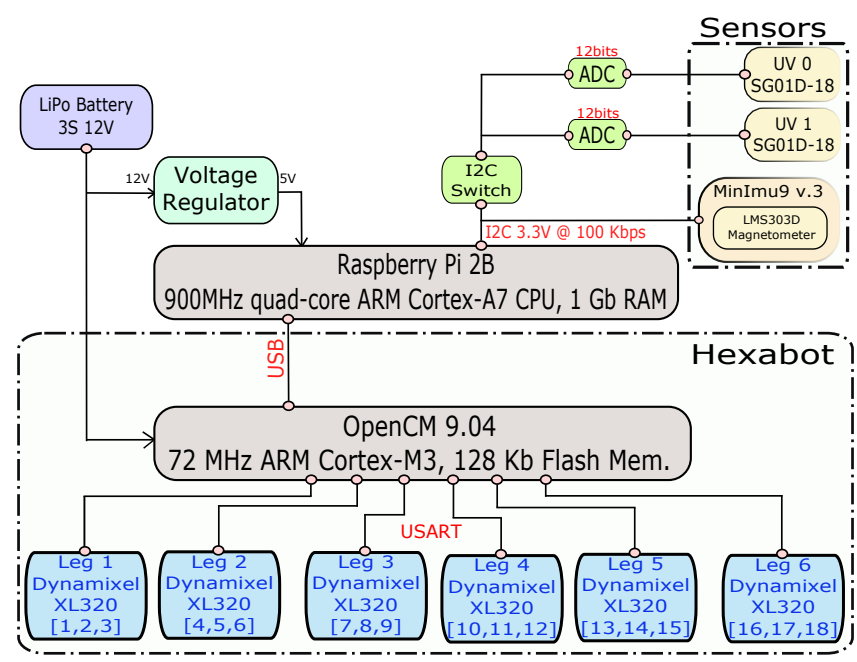

Fig. 4. Robot electronic architecture. The dashed line marks out the robot controller and actuators. The magnetometer and UV-polarized light sensors (in yellow) are connected to the Raspberry Pi 2B board using I2C communication protocol.

ARM Cortex-A7) which achieves sensor data acquisition and processing, and send high level orders the robot controller. The celestial compass is embedded on the dorsal part of the robot (see figure 1.A). Communication with the Raspberry Pi board was implemented by means of I2C protocol (figure 4).

\section{EXPERIMENTS}

Hexabot was set to tripod gait for all navigational tasks as it provides an optimal compromise between high walking speed and moderate attitude disturbances. However, interactions between legs and the ground tend to cause a large drift of the trajectory. We propose here to use our UVpolarized light compass to contain the drift occurring after each stride. All experiments were done between 02/02/2017 and $02 / 20 / 2017$ in outdoor conditions, at any time of the day, and were located in an open-air car park in the Luminy campus $\left(43^{\circ} 14^{\prime} 01.6^{\prime \prime} N ; 5^{\circ} 26^{\prime} 39.2^{\prime \prime} E\right)$ of Aix-Marseille Université, Marseille, France. The angular precision of the UVlight polarized compass was arbitrarily set at $1.29^{\circ}$ for all experiments. The acquisition time was consequently of $42 s$.

Drift measurements were made for five outdoor straightforward walking tasks on a flat but rough terrain. Tests were conducted over six seconds at maximum walking speed. Results show an average heading direction disturbance of $28^{\circ}$ (magnetometer measurements). In those conditions, Hexabot drifts from initial walking axis by an average length of one meter.

\section{A. Recovery of orientation under various weather conditions}

The ability to reorientate Hexabot after a random yaw disturbance was first tested. The reorientation tasks consists of : (1) the robot acquires its initial heading direction with the celestial compass; (2) the robot turns by a random angle and then acquires its new heading direction; (3) the robot computes the difference between the initial and the new heading direction and uses it as an order to turn back to its initial orientation. Finally, we compare the ground truth measurements (magnetometer ${ }^{4}$ ) before the disturbance and after the angular correction. To prevent any yaw ambiguity, disturbances were set between $-70^{\circ}$ and $+70^{\circ}$. Such a restriction makes sense as angular drifts over a straightforward walking task are systematically small (less than $30^{\circ}$ over a 3-meter walk) but randomly oriented in the both directions due to interactions with the ground.

Figure 5 shows heading errors under three different weather conditions. The variability of performances in orientation recovery can be explained by the non-regularity of Hexabot's stride length while turning due to interactions with the ground. In view of this, the presented results exhibit great performance, especially under clear sky conditions. The decrease noticed under cloudy sky and overcast sky conditions stems from the low degree of polarization of the skylight [6] which implies the overpowering of the Rayleigh scattering, thus disturbing the polarization pattern of the skylight.

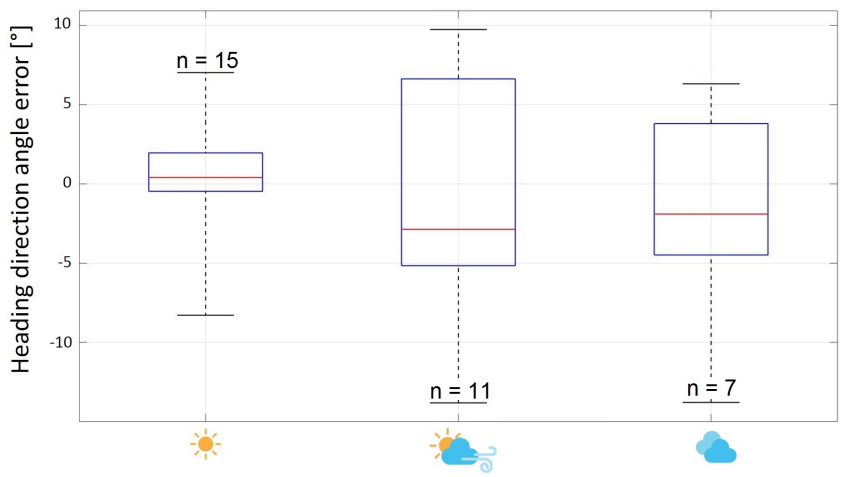

Fig. 5. Heading direction angle errors in degrees in function of weather conditions. From left to right, the median heading direction angle error measured is equal to $0.4^{\circ},-2.9^{\circ}$, and $-1.9^{\circ}$. UV-index from 1 to 2 (source: French Meteorological services).

Considering the results obtained with Sahabot robot in [10], our UV-polarized light compass provides similar and even slightly better results under clear sky, and promising results under bad meteorological conditions such as clouds in the sky and a much lower level of UV radiance due to the period and the location of experiments.

\section{B. Heading-lock over a straight-forward walking task}

As mentioned above, Hexabot exhibits important drifts in yaw orientation and the celestial compass successfully corrected yaw disturbances at fix point. We now test this ability to maintain the robot within a straight-forward trajectory applying yaw correction after each walking step. First, the initial angle is acquired. Then Hexabot executes a series of strides during two seconds and measures its new yaw orientation, the value of which is compared to the initial one to compute the yaw angle correction to be applied. Finally, Hexabot executes the corresponding turning

\footnotetext{
${ }^{4}$ Experiments were conducted with a calibrated magnetometer and away from any magnetic fields interference.
} 


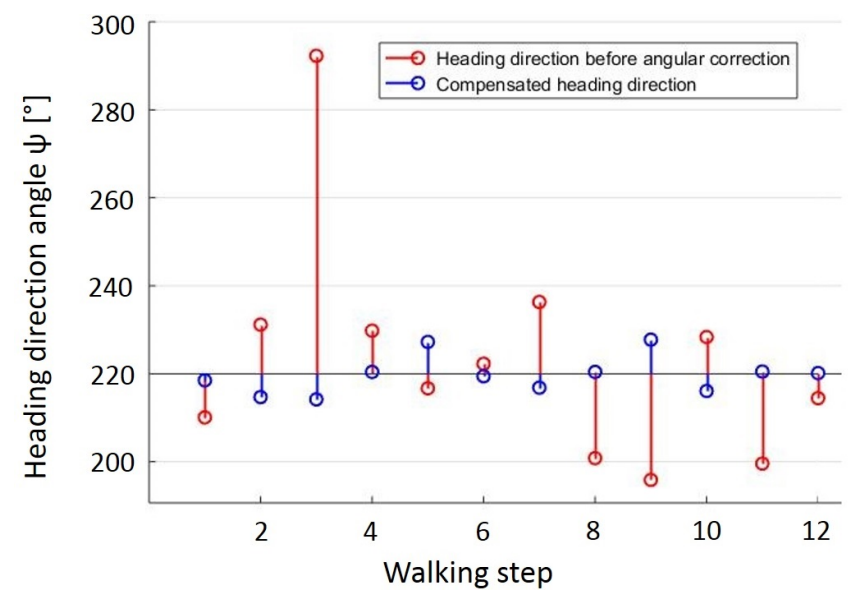

Fig. 6. Evolution of the measured heading direction angle $\psi$ before (in red) and after (in blue) angular correction using only the UV-polarized light compass during a straight-forward walking task. Walking steps measurements from 1 to 6 were acquired on 02/18/2017 while the next six measurements were acquired on $02 / 20 / 2017$.

movement before moving to the next series of strides. Due to power supply limits and to avoid the impact of polarization shifts induced by the sun movements, data were acquired over two distinct days (02/18/2017 and 02/20/2017), but experiments were all performed at the same time $(2: 00 \mathrm{pm})$ under perfectly clear sky conditions with a UV index of 2.

Results for all experiments are shown in figure 6 . The average heading angle error measured is of $-0.3^{\circ}$ which is consistent with performances exhibited previously under clear sky conditions. The peak error measured is of $7.7^{\circ}$, occurring during the ninth walking step. Since there were no clouds in the sky, the polarization pattern remained rather constant all over the experiments. As a consequence, the heading direction error is mainly caused by interactions between legs and the ground.

\section{CONCLUSION}

In this paper, a novel insect-inspired celestial compass was introduced. Performances analysis during winter and spring showed this compass can be used in all weather conditions, including high and low UV-index, clear and covered sky. The sensor was then embedded onto an ant-inspired walking robot to maintain the robot's heading direction constant while walking.

The heading recovery experiments performed both at fix point and during a walking task showed highly precise and reliable results under clear-sky conditions, with an average steady state error as small as $0.3^{\circ}$. Results under cloudy-sky conditions also exhibits good performances, from $0.8^{\circ}$ under variable weather, to $1.9^{\circ}$ under overcast sky, but slightly less reliable due to the high variability of meteorological conditions.

Future work will focus on the impact of the turning uncertainty of the robot on the heading direction. Residual heading-lock errors can be reduced by changing to a closedloop system showing the suitability of this new optical compass sensor for autonomous robotic tasks such as homing.

\section{ACKNOWLEDGMENT}

The authors would like to thank Grégoire Passault and Olivier Ly for their investment to the maintenance of the hexapod robot.

\section{REFERENCES}

[1] E. Bergamini, G. Ligorio, A. Summa, G. Vannozzi, A. Cappozzo, and A. Sabatini. Estimating orientation using magnetic and inertial sensors and different sensor fusion approaches: accuracy assessment in manual and locomotion tasks, Sensors, vol. 14, no 10, pp. 18625-18649, 2014.

[2] R. Wehner, B. Michel, and P. Antonsen, Visual navigation in insects: coupling of egocentric and geocentric information, Journal of Experimental Biology, vol. 199, pp. 129-140, 1996.

[3] K.L. Coulson, Polarization and Intensity of Light in the Atmosphere, A Deepak Pub, 1988.

[4] T. Labhart, and E.P. Meyer, Detectors for polarized skylight in insects: a survey of ommatidial specializations in the dorsal rim area of the compound eye, Microscopy research and technique, vol. 47, no 6, pp. 368-379, 1999

[5] T. Labhart, Polarization-opponent interneurons in the insect visual system, Nature, vol. 331, no 6155, pp. 435-437, 1988.

[6] M.L. Brines, and J.L. Gould, Skylight polarization patterns and animal orientation, J. exp. Biol, vol. 96, pp. 69-91, 1982.

[7] A. Barta, and G. Horváth, Why is it advantageous for animals to detect celestial polarization in the ultraviolet? Skylight polarization under clouds and canopies is strongest in the UV, Journal of Theoretical Biology, vol. 226, no 4, pp. 429-437, 2004.

[8] S. Heinze, and U. Homberg, Linking the input to the output: new sets of neurons complement the polarization vision network in the locust central complex, Journal of Neuroscience, vol. 29, no 15, pp. 49114921, 2009

[9] R. Wehner, Desert ant navigation: how miniature brains solve complex tasks, Journal of Comparative Physiology A, vol. 189, no 8, pp. 579588, 2003.

[10] D. Lambrinos, H. Kobayashi, R. Pfeifer, M. Maris, T. Labhart and R. Wehner, An autonomous agent navigating with a polarized light compass, Adaptive Behavior, vol. 6, pp. 131-161, 1997.

[11] D. Lambrinos, R. Möller, T. Labhart, R. Pfeifer, and R. Wehner, A mobile robot employing insect strategies for navigation, Robotics and Autonomous systems, vol. 30, pp. 39-64, 2000.

[12] R. Wehner, and S. Wehner, Insect navigation: use of maps or Ariadne's thread?, Ethology Ecology \& Evolution, vol. 2, no 1, pp. 27-48, 1990.

[13] J. Chu, K. Zhao, Q. Zhang, and T. Wang, Construction and performance test of a novel polarization sensor for navigation, Sensors and Actuators A: Physical, vol. 148, no 1, pp. 75-82, 2008.

[14] J. Chu, H. Wang, W. Chen, and R. Li, Application of a novel polarization sensor to mobile robot navigation, Mechatronics and Automation, 2009, ICMA 2009, International Conference on. IEEE, pp. 3763-3768, 2009.

[15] J. Chu, Z. Wang, L. Guan, Z. Liu, Y. Wang, and R. Zhang, Integrated polarization dependent photodetector and its application for polarization navigation, IEEE Photonics Technol. Lett, vol. 26, no 5, p. 469472, 2014.

[16] J. Chahl, and A. Mizutani, Biomimetic attitude and orientation sensors, IEEE Sensors Journal, vol. 12, no 2, pp. 289-297, 2012.

[17] G. Passault, Q. Rouxel, F. Petit, and O. Ly, Metabot: a low-cost legged robotics platform for education, In : Autonomous Robot Systems and Competitions (ICARSC), 2016 International Conference on. IEEE, pp. 283-287, 2016.

[18] J. Dupeyroux, G. Passault, F. Ruffier, S. Viollet, and J. Serres, Hexabot: a small 3D-printed six-legged walking robot designed for desert ant-like navigation tasks, 20th World Congress of the International Federation of Automatic Control, Toulouse, France, in press. 